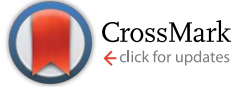

Cite this: RSC Adv., 2015, 5, 53054

Received 23rd May 2015

Accepted 10th June 2015

DOI: 10.1039/c5ra09708h

www.rsc.org/advances

\section{Enhancement of dielectric permittivity by incorporating PDMS-PEG multiblock copolymers in silicone elastomers $\uparrow$}

\begin{abstract}
Aliff Hisyam A. Razak, ${ }^{\text {ab }}$ Peter Szabo a and Anne Ladegaard Skov ${ }^{\star a}$
A silicone elastomer from PDMS-PEG multiblock copolymer has been prepared by use of silylation reactions for both copolymer preparation and crosslinking. The dielectric and mechanical properties of the silicone elastomers were carefully investigated, as well as the morphology of the elastomers was investigated by SEM. The developed silicone elastomers were too conductive to be utilized as dielectric elastomers but it was shown that when the above silicone elastomers were mixed with a commercial silicone elastomer, the resulting elastomer had very favourable properties for dielectric elastomers due to a significantly increased dielectric permittivity. The conductivity also remained low due to the resulting discontinuity in PEG within the silicone matrix.
\end{abstract}

\section{Introduction}

Dielectric elastomers (DEs) have been studied extensively with respect to finding both new and better elastomer candidates and novel applications. ${ }^{1-4}$ DEs are elastomers which exhibit a change in size or shape when stimulated by an external electric field. They are also known as "compliant capacitors", with actuation occurring when electrostatic stress exceeds elastic stress. ${ }^{5}$ Such properties have enabled DEs to play a significant role in applications as actuators, sensors and generators.

Dielectric elastomers with high relative permittivity possess high electrical energy in the form of charge separation, due to polarisation. In an unactuated state, the elastomer can withstand a given electrical field, the so-called electrical "breakdown strength",' but above this electrical field the DE will short-circuit. Another common failure associated with DEs is electromechanical instability (EMI), which arises during actuation when attractive forces between the two electrodes become dominant and locally exceed a certain threshold value that cannot be balanced by the material's resistance to compression. ${ }^{7,8}$ This phenomenon, which is also known as

${ }^{a}$ Danish Polymer Center, Department of Chemical and Biochemical Engineering, Technical University of Denmark, Building 229, 2800 Kgs. Lyngby, Denmark. E-mail: al@kt.dtu.dk

${ }^{b}$ Department of Chemical Engineering Technology, Faculty of Engineering Technology, University of Tun Hussein Onn Malaysia, 86400 Parit Raja, Batu Pahat, Johor, Malaysia

$\dagger$ Electronic supplementary information (ESI) available: ${ }^{1} \mathrm{H}-\mathrm{NMR}$ spectra and contact angles of the synthesised PDMS-PEG multiblock copolymers (PDMS81-PEG, PDMS14-PEG, PDMS7-PEG and PDMS3-PEG). Results of dielectric and rheology for binary polymer blends by incorporating PDMS-PEG multiblock copolymers at different wt\% $(5,10,15$ and 20) into a PDMS network (MJK). See DOI: 10.1039/c5ra09708h "electromechanical breakdown", can usually be eliminated by prestretching the elastomer, since prestretching has a combined effect of hardening the silicone elastomer, decreasing film thickness and increasing electrical breakdown strength. ${ }^{9,10}$

Polydimethylsiloxanes (PDMS), as one promising type of dielectric elastomer, exhibit large ultimate extension. ${ }^{\mathbf{1 1 - 1 4}}$ Despite its significant deformation, the drawback of PDMS is that it has low permittivity, in relation to the net dipole moment $(\mu)$, of 0.6-0.9 D. ${ }^{15}$ On the positive side, PDMS is known to have very low conductivity. ${ }^{16}$ In contrast, polyethyleneglycols (PEG) show high permittivity as a result of a dipole moment of $3.91 \mathrm{D},{ }^{17}$ yet they are incapable of actuating, as they are highly conductive. ${ }^{18}$ Combining PDMS and PEG as a block copolymer presents the possibility of substantially improving properties such as high permittivity and non-conductivity, whereby PEG enhances permittivity and PDMS facilitates actuation through its non-conductive nature and inherent softness. The synthesis of the PDMS-PEG multiblock copolymer utilised herein is based on hydrosilylation, as shown in Fig. 1.

An astonishing feature of block copolymers is the variety of morphologies due to self-assembly in bulk or in solution. ${ }^{\mathbf{1 9 , 2 0}}$ In principle, a diblock copolymer, which is the simplest block copolymer, assembles into different morphologies, such as sphere (S), cylinder (C), gyroid (G) and lamellar (L). ${ }^{19,21}$ These morphologies can be achieved when two immiscible, covalentlybonded polymers microphase separately. ${ }^{22}$ These morphologies can be changed by varying the volume fraction of one constituent in the diblock copolymer. For triblock copolymers, the morphologies are more complex, mainly due to the sequence order of three distinct polymers, e.g. ABC, ACB, BAC and BCA, which introduces further degrees of freedom and thus allows for the assembly of nearly 30 different morphologies. ${ }^{19}$ The 


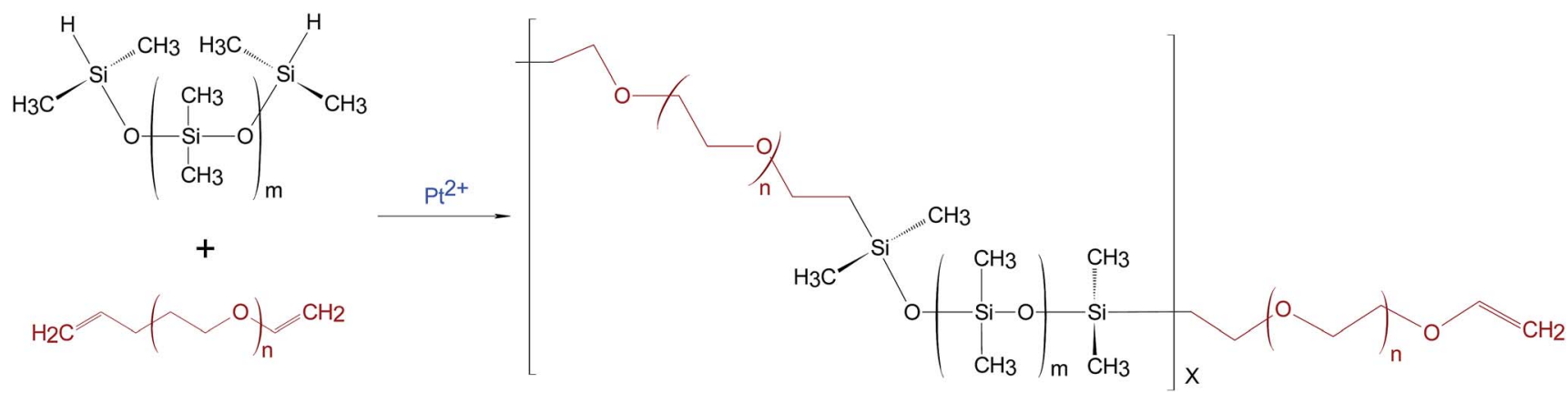

Fig. 1 The hydrosilylation reaction utilized when preparing a PDMS-PEG multiblock copolymer, where $m$ is the number of repeating dimethylsiloxane units in PDMS, and $n=4$ is the constant number of repeating ethyleneglycol units in PEG. $X$ is the number of repeating PDMS-PEG units in multiblock copolymers.

similarity shared by the block copolymers is that they have four common equilibrium morphologies (S, C, G and L). ${ }^{21}$

Here, elastomers are prepared by means of phase separating PDMS-PEG multiblock copolymers, whereby the copolymers' blocks are expected to segregate to form well-defined structures, depending on the chain lengths of the two constituents. Subsequently the phase-separated copolymers are cross-linked via silylation into elastomers.

\section{Experimental}

\section{Materials and reagents}

Hydride-terminated polydimethylsiloxanes (H-PDMS) used in the synthesis of the PDMS-PEG multiblock copolymer were DMS-H21, DMS-H11, DMS-H03 and SIH6117.0, each with an average molecular weight $\left(M_{\mathrm{n}}\right)$ of $6000,1050,550$ and 208 $\mathrm{g} \mathrm{mol}^{-1}$, respectively. They were purchased from Gelest Inc., while polyethyleneglycol divinyl ether (PEG-DE) was acquired from Sigma Aldrich. A commercial PDMS elastomer [MJK 4/13] was obtained from Wacker Chemie AG, and platinum-divinyltetramethyl disiloxane complex [SIP6830.3] was purchased from Gelest Inc. and contained $3.25 \%$ of platinum in xylene. A hydride-terminated methyl-hydrosiloxane-dimethylsiloxane copolymer [HMS-501] ( $M_{\mathrm{n}}$ of $1050 \mathrm{~g} \mathrm{~mol} \mathrm{~m}^{-1}$, 9-functional) cross-linker, along with tetravinyltetramethyl-cyclotetrasiloxane [SIT-7900] as an inhibitor, was purchased from Gelest Inc. Both methanol and toluene were purchased from Sigma Aldrich.

\section{Synthesis of the PDMS-PEG prepolymer}

The procedure used to synthesise PDMS-PEG multiblock copolymer was amended from that employed by Klasner et al. ${ }^{23}$ and Jukarainen et al. ${ }^{24}$ All glassware was thoroughly cleaned and dried at a temperature of $200^{\circ} \mathrm{C}$. The characterisations on $M_{\mathrm{n}}$ of DMS-H21, DMS-H11, DMS-H03, SIH6117.0 and PEG-DE were performed using ${ }^{1} \mathrm{H}$-NMR to obtain precise $M_{\mathrm{n}}$ for the stoichiometry calculations.

The theoretical PDMS-PEG repeating units in the multiblock copolymer were calculated from a target molecular weight of $30 \mathrm{~kg} \mathrm{~mol}^{-1}$, whereby the number of blocks for PDMS and PEG were $X$ and $(X+1)$, respectively:

$$
X=\frac{30000-M_{\mathrm{n}, \mathrm{PEG}}}{M_{\mathrm{n}, \mathrm{PDMS}}+M_{\mathrm{n}, \mathrm{PEG}}}
$$

where $M_{\mathrm{n}, \mathrm{PDMS}}$ and $M_{\mathrm{n}, \mathrm{PEG}}$ are the molecular weight of PDMS and PEG, respectively.

The stoichiometric ratio for preparing multiblock copolymers $\left(r_{1}\right)$ was calculated as:

$$
r_{1}=\frac{[\text { vinyl }]}{[\text { hydride }]}=\frac{(X+1) f_{\mathrm{PEG}-\mathrm{DE}}}{X f_{\mathrm{H}-\mathrm{PDMS}}}=\frac{X+1}{X}
$$

where $f_{\text {PEG-DE }}$ and $f_{\text {H-PDMs }}$ are the functionality of PEG-DE and $\mathrm{H}$-PDMS, respectively. ${ }^{25}$ Both polymers in this case were difunctional $(f=2)$, and the telechelic vinyl groups of the resulting copolymer were targeted.

Dry toluene (prepared by molecular sieving) was added into the flask at $30 \mathrm{wt} \%$ of the total mass of H-PDMS and PEG-DE. The initial concentration of the platinum catalyst was 3120 parts per million (ppm). From this solution, the amount of catalyst solution was determined, in order to obtain a final concentration of $30 \mathrm{ppm}$ in the reaction mixture, by assuming the density of the mixture was $1 \mathrm{~g} \mathrm{~cm}^{-3}$. The reaction occurred at $60{ }^{\circ} \mathrm{C}$ with mild stirring and in the presence of nitrogen gas to eliminate air inside the flask. The duration of the hydrosilylation reaction depended on the chain length of H-PDMS and ranged from 2 to 6 hours. The disappearance of a $\mathrm{Si}-\mathrm{H}$ bond signal at $4.70 \mathrm{ppm}$ was checked by H-NMR, to ensure that all hydrides in the PDMS had been fully consumed during the reaction; refer to ESI for NMR spectra in Fig. S1a-d. $\uparrow$ The final solution was viscous and appeared light bronze in colour. Any remaining solvent (toluene) was removed with a rotary evaporator for a couple of hours. The product was purified by cold methanol precipitation, in order to remove excess PEG-DE, and washing was repeated at least five times. Methanol from the precipitation process was excluded by using a rotary evaporator for a few hours and then placing the mixture in a vacuum for a day.

\section{Experimental setup for the PDMS-PEG block copolymer}

To distinguish the PDMS-PEG multiblock copolymer samples from different PDMS volume fractions, they were named based on four different repeating unit numbers in the constituent 
Table 1 Sample details for PDMS-PEG multiblock copolymers ${ }^{a}$

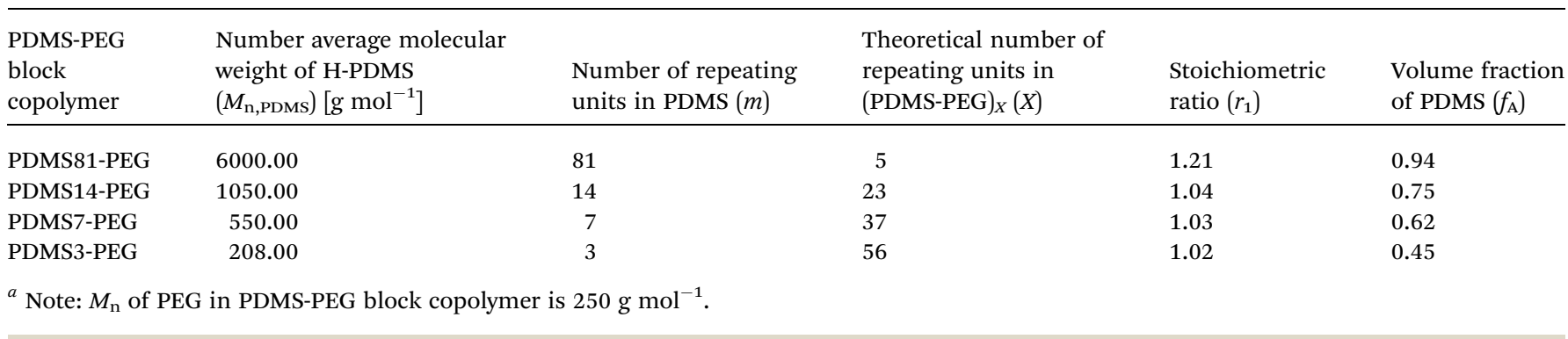

polymer, as listed in Table 1. Asymmetrical morphologies in the PDMS-PEG multiblock copolymer were obtained by varying PDMS chain lengths ( $m=3,7,14,81$ ) while sustaining the equivalent PEG chain length $(n=4)$, which in turn produced PDMS3-PEG, PDMS7-PEG, PDMS14-PEG and PDMS81-PEG, respectively. Hence PDMS81-PEG constituted the highest volume fraction of PDMS in the block copolymer (0.94), whereas the lowest volume fraction produced in this study was 0.45 (belonging to PDMS3-PEG).

\section{Binary polymer blends (BPBs)}

PDMS-PEG multiblock copolymers were incorporated into PDMS elastomer (MJK) at 5, 10, 15 and $20 \mathrm{wt} \%$. All mixtures were speedmixed at $3500 \mathrm{rpm}$ for 2 minutes. After that, the blends were immediately cross-linked. The blends produced 16 samples in total.

\section{Cross-linking}

Four samples of PDMS-PEG multiblock copolymers and 16 samples of BPB were prepared. The stoichiometric ratio for the cross-linking $\left(r_{2}\right)$ was calculated as:

$$
r_{2}=\frac{[\text { hydride }]}{[\text { vinyl }]}=\frac{f_{\mathrm{HMS}}[\mathrm{HMS}]}{f_{\mathrm{BCP}}[\mathrm{BCP}]}
$$

where $f_{\mathrm{HMS}}$ and $f_{\mathrm{BCP}}$ were the numbers of the HMS-501 (9-functional) functional group and the PDMS-PEG block copolymer (2-functional), respectively, while [...] indicates the initial concentration. ${ }^{26,27}$

The values of $r_{2}$ were calculated based on the mass of PDMSPEG prepolymers added into the blends. The inhibitor (SIT7900) and the platinum catalyst were added to the blends at $1 \mathrm{wt} \%$ and $30 \mathrm{ppm}$, respectively. Those blends which consisted of PDMS-PEG prepolymer, namely MJK4/13, SIT7900 and 30 ppm platinum catalyst, were speed-mixed rigorously at $3000 \mathrm{rpm}$ for 2 minutes. Cross-linker (HMS-501) was added, and the resulting mixture was additionally speed-mixed at $1500 \mathrm{rpm}$ for 2 minutes. The cross-linked films were cured at a temperature of $60{ }^{\circ} \mathrm{C}$ overnight and then subsequently post-cured at $110{ }^{\circ} \mathrm{C}$ for 2 hours.

\section{Characterisations}

The NMR equipment utilised in this instance was the Bruker $300 \mathrm{MHz}$ NMR. The number of scannings per sample was 128.
The sample was prepared by diluting $50 \mathrm{mg}$ of the sample in $0.5 \mathrm{~mL}$ of deuterated chloroform $\left(\mathrm{CDCl}_{3}\right)$.

Static contact angles, created by using the "sessile dropneedle in" method, were taken at a room temperature of $23{ }^{\circ} \mathrm{C}$ using Dataphysics OCA20. The contact angle was measured by dropping $6 \mu \mathrm{L}$ of deionised water onto the PDMS-PEG multiblock copolymer and BPB films. Measurements for each contact angle were taken for 65 seconds, and the contact angles were analysed every 5 seconds, in order to obtain contact angle versus time profiles.

Linear viscoelasticity (LVE) properties, i.e. storage and loss moduli, were characterised at room temperature using TA Instruments' ARES-G2. The geometry of the parallel plate was $25 \mathrm{~mm}$. The axial force, strain and frequency ranges were $5 \mathrm{~N}$, $2 \%$ and $100-0.01 \mathrm{~Hz}$, respectively. The Young's modulus can be determined as $Y=2(1+\nu) G=3 G$, since Poisson's ratio $(\nu)$ is close to 0.5 , due to the incompressibility of silicones.

Dielectric permittivity, loss permittivity and conductivity were measured at a frequency of $10^{6}$ to $10^{-1}$ using a broadband dielectric spectrometer from Novocontrol Technologies $\mathrm{GmbH}$ \& Co. KG, Germany. The electrode diameter was $20 \mathrm{~mm}$.

The breakdown tests were carried out on an in-house-built device based on international standards (IEC 60243-1 (1998) and IEC 60243-2 (2001)). ${ }^{28}$ Samples with a film thickness less than $100 \mu \mathrm{m}$ were used, as breakdown strength depends greatly on sample thickness. ${ }^{10}$ The film was slid between the two spherical electrodes (radius of $20 \mathrm{~mm}$ ), and breakdown was measured at the point of contact, with a stepwise increasing voltage applied (50 to $100 \mathrm{~V}$ per step) at a rate of $0.5-1$ steps per $\mathrm{s}^{29}$ Each sample was measured up to 12 times, and the average of these values was then taken as the breakdown strength.

The SEM model, FEI Inspect $\mathrm{S}$, used to characterise nanoscale images, performed energy-dispersive X-ray and wavelength dispersive measurements. The accelerating voltage and resolution were $200 \mathrm{~V}$ to $30 \mathrm{kV}$ and $50 \mathrm{~nm}$ at $30 \mathrm{kV}$, respectively, while the imaging modes used high and low vacuums.

The number average molecular weight $\left(M_{\mathrm{n}}\right)$ determinations for PDMS-PEG multiblock copolymers were performed on an SEC instrument consisting of a Viscotek GPCmax VE-2001 instrument equipped with a Viscotek TriSEC Model 302 triple detector using two PLgel mixed-D columns from Polymer Laboratories. Samples were run in tetrahydrofuran (THF) at $30{ }^{\circ} \mathrm{C}$ and at a rate of $1 \mathrm{~mL} \mathrm{~min}^{-1}$. Molar mass characteristics were calculated using polydimethylsiloxane standards. 


\section{Results and discussion}

\section{PDMS-PEG multiblock copolymer}

The PDMS-PEG block copolymer samples with different PDMS chain-lengths were characterised by means of sizeexclusion chromatography (SEC), while the cross-linked samples were analysed by means of dielectric spectroscopy and rheology. Results for the average number of molecular weights obtained from SEC, shown in Table 2, indicate that synthesised PDMS-PEG multiblock copolymers possess lower $M_{\mathrm{n}}$ than targeted.

The relative permittivity of the multiblock copolymers is shown in Fig. 2. Relative permittivity for the copolymer with the least PEG (PDMS81-PEG) is constant at all frequencies, with a slight increase at low frequencies. This behaviour is similar to that of the reference elastomer (MJK), but the PDMS81-PEG multiblock copolymer has three-fold higher relative permittivity. For samples with higher PEG content, significant relaxation takes place at low frequencies, leading to increased permittivity (as seen in Fig. 3), while dielectric loss also increases very abruptly when decreasing the frequency. This behaviour indicates conductive nature of the elastomers. In Fig. 4 the conductivity of the copolymers is shown. It is obvious that they are all conductive, due to the display of a plateau in conductivity at low frequencies. The block copolymers have conductivities of the order of $10^{2}$ to $10^{5}$ higher than those of the reference elastomer (MJK).

Table 2 Average number of multiblock copolymers molecular weights

\begin{tabular}{ll}
$\begin{array}{l}\text { PDMS-PEG block } \\
\text { copolymer }\end{array}$ & $\begin{array}{l}\text { Experimental } M_{\mathrm{n}, \mathrm{T}} \\
\left(10^{3} \mathrm{~g} \mathrm{~mol}^{-1}\right)\end{array}$ \\
\hline PDMS81-PEG & 13 \\
PDMS14-PEG & 2.5 \\
PDMS7-PEG & 3.6 \\
PDMS3-PEG & 1.2
\end{tabular}

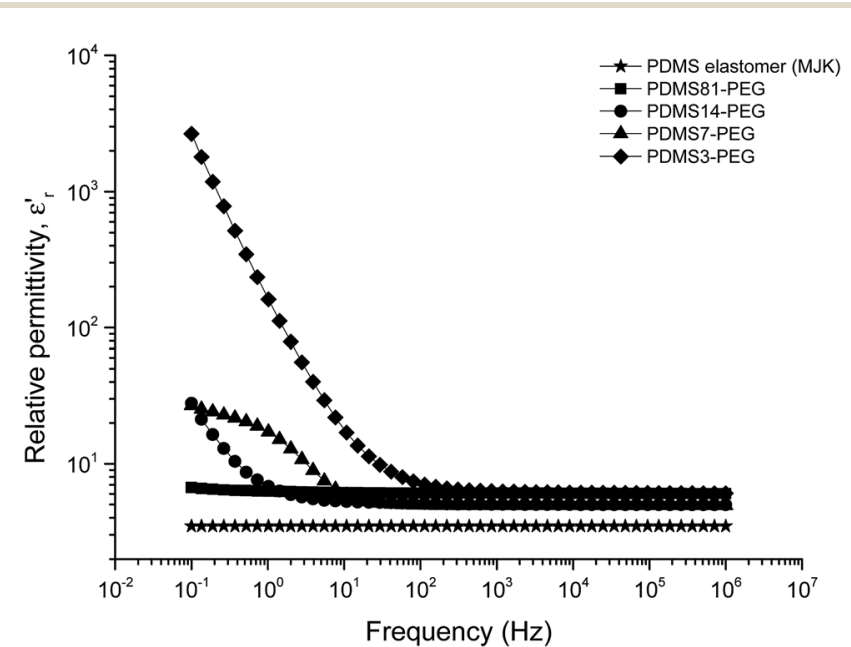

Fig. 2 Relative permittivity of cross-linked PDMS-PEG multiblock copolymers at $23^{\circ} \mathrm{C}$.

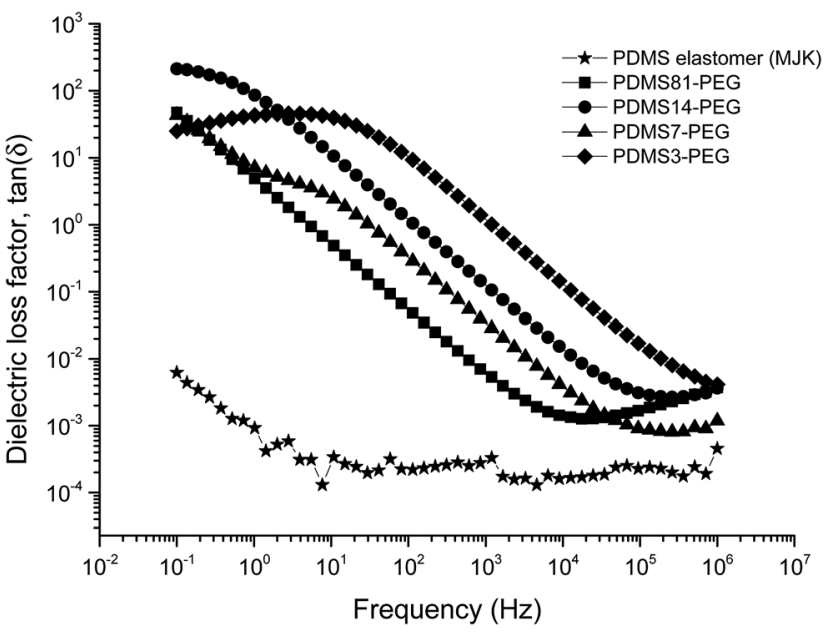

Fig. 3 Dielectric loss factor for cross-linked PDMS-PEG multiblock copolymers at $23^{\circ} \mathrm{C}$.

The rheological properties of the cross-linked copolymers are shown in Fig. 5. The PDMS14-PEG and PDMS81-PEG samples show the behaviour of very soft networks with low storage moduli compared to silicone elastomers, and they also demonstrate significant relaxation at low frequencies, which further indicates the inherent softness. In contrast, the PDMS3-PEG and PDMS7-PEG samples possess PEG-like properties with high storage moduli and low losses. Furthermore, their shear modulus is higher than that of the reinforced commercial silicone elastomer. Therefore, it is clear that an increase of PEG constituents in a PDMS-PEG multiblock copolymer reinforces the network comparable with the effect of silica fillers. It is noteworthy that PDMS81-PEG and PDMS14-PEG closely resemble each other despite PDMS81-PEG being significantly shorter than PDMS14-PEG (see Table 2), and thus PDMS81-PEG should provide significantly higher crosslink density and thus higher $G$. However, this effect cannot be seen simply because the increased content of PEG in PDMS14-PEG has an identical cross-linking effect.

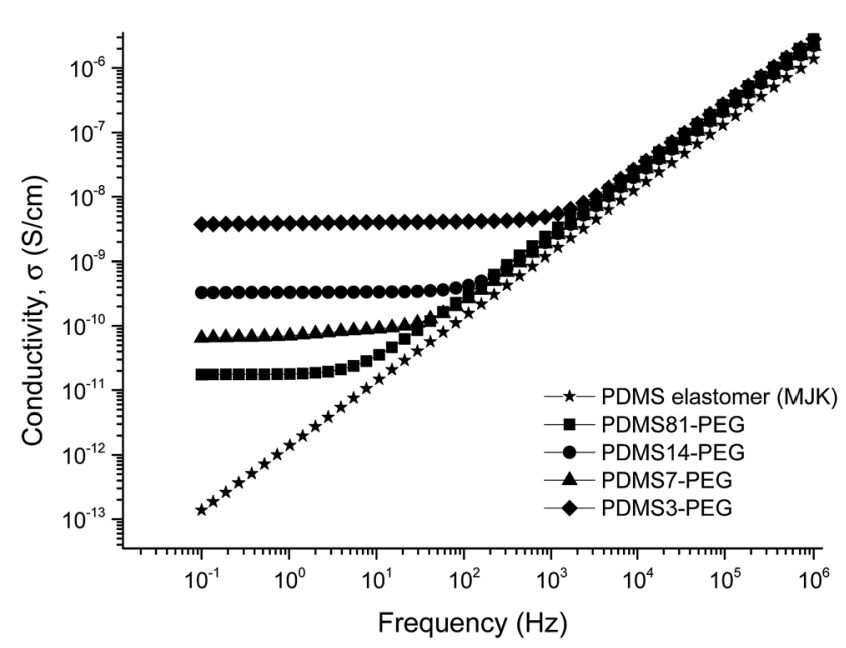

Fig. 4 Conductivity of PDMS-PEG multiblock copolymers at $23^{\circ} \mathrm{C}$. 


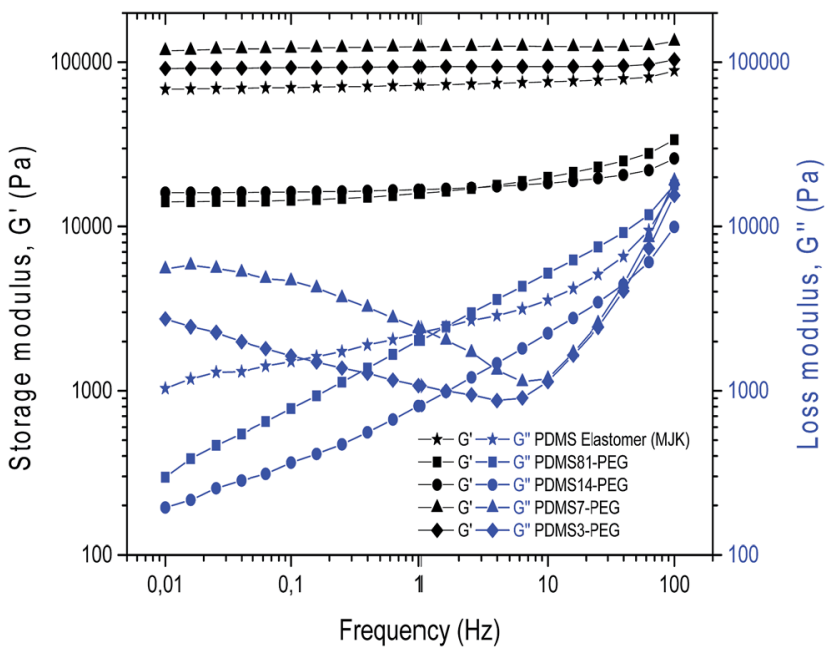

Fig. 5 Comparison between the storage and loss modulus PDMS-PEG multiblock copolymers at $23^{\circ} \mathrm{C}$.

Binary polymer block copolymer and silicone elastomer blends

Due to the conductivity of PDMS-PEG multiblock copolymers, they were further blended and cross-linked into a commercial PDMS elastomer (MJK). Incorporating the block copolymers into a silicone network as a binary polymer blend (BPB) can facilitate the creation of PEG spheres, as illustrated in Fig. 6. The blends consist of PDMS-PEG multiblock copolymers at loadings of 5, 10, 15 and $20 \mathrm{wt} \%$ and are denoted as MJK/PDMS $i$, where $i=81,14$, 7,3 . When increasing PEG fractions, unfavourable and discontinuous morphologies may be formed.

\section{Dielectric properties of the binary polymer blends}

The relative dielectric permittivity and loss permittivity of the polymer blends are shown in Fig. 7 and 8. Relative permittivities are significantly improved compared to the reference elastomer (MJK), and loss permittivities are substantially lower than those of the pure copolymers - as hypothesised. Refer to ESI Fig. S2-4† for data for all samples.

In general, the storage permittivity of MJK/PDMS7 increases as the wt\% of the PDMS7-PEG multiblock copolymer increases

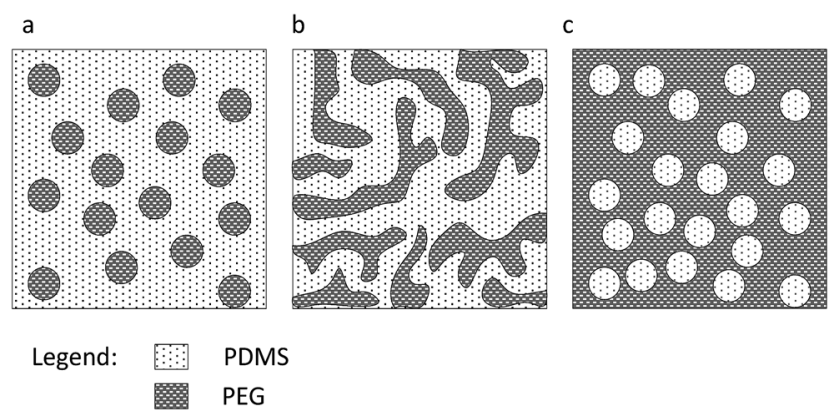

Fig. 6 Illustration of morphologies for BPB of PDMS-PEG block copolymer and silicone elastomer: (a) continuous phase in PDMS (b). Co-continuous phase in PDMS (c). Discontinuous phase in PDMS.

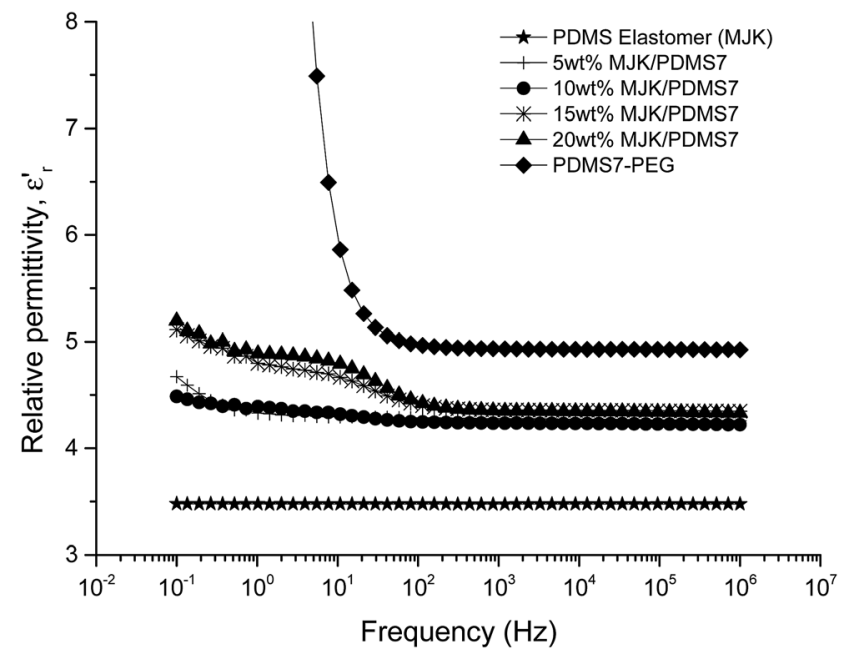

Fig. 7 The relative permittivity of MJK/PDMS7 (5-20 wt\% of PDMS7-PEG) at $23^{\circ} \mathrm{C}$.

in line with loadings from 5 to $20 \mathrm{wt} \%$. Incorporating $20 \mathrm{wt} \%$ of PDMS7-PEG in a PDMS network yields the highest relative permittivity (5.2), which is an increase of $60 \%$ compared to the relative permittivity of MJK (3.5). The small increase in relative permittivity at low frequencies for MJK/PDMS7, with 5 and $10 \mathrm{wt} \%$, is due to electrode polarisation effects occurring during the measurement process. However, this can be corrected by applying silicone grease between the sample and the electrode. $^{30}$ The dynamic dipole orientation of polymer molecules resulting from polarisation are observed for MJK/PDMS7 at 15 and $20 \mathrm{wt} \%$, as Debye-relaxation peaks occur at frequencies of $10^{0}$ to $10^{3} \mathrm{~Hz}$.

One essential finding from the dielectric characterisation is that none of the polymer blends is conductive. To further analyse the optimum polymer blend, selection based on the sample which gives the lowest dielectric loss factor is carried out. Polymer blends of MJK/PDMS3, MJK/PDMS14 and

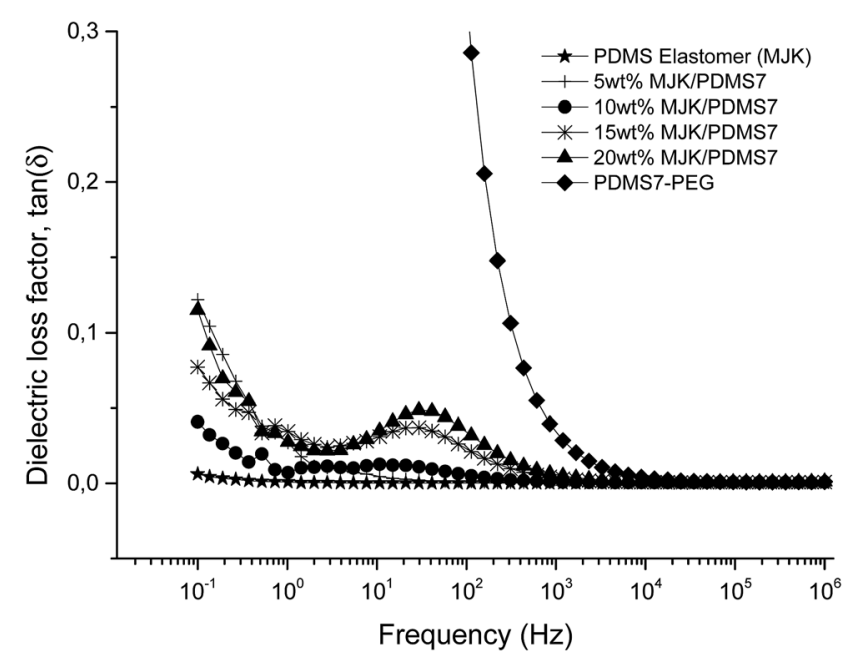

Fig. 8 The dielectric loss factor of MJK/PDMS7 (5-20 wt\% of PDMS7-PEG) at $23^{\circ} \mathrm{C}$. 


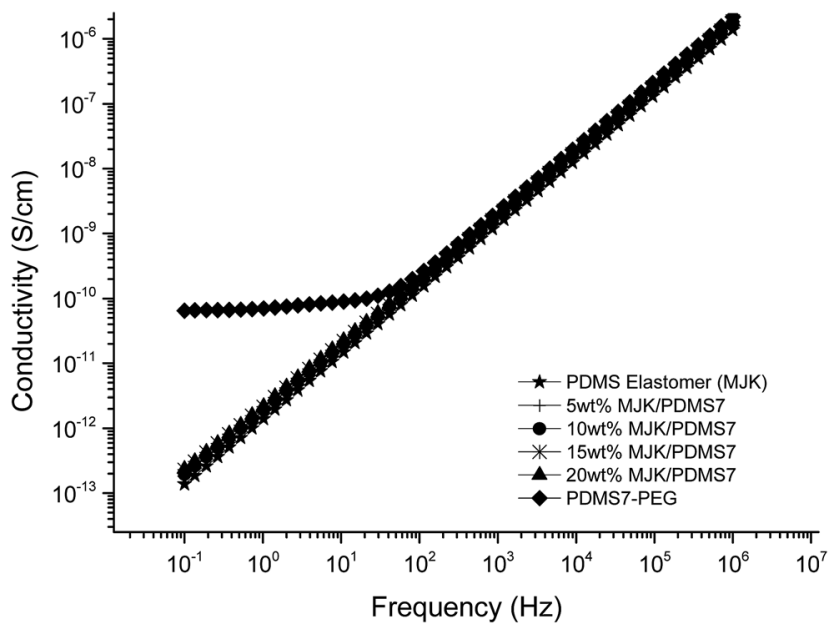

Fig. 9 The conductivity of MJK/PDMS7 (5-20 wt\% of PDMS7-PEG) at $23^{\circ} \mathrm{C}$.

MJK/PDMS81 possess electrical loss factors in the ranges of $0.5-0.9,0.25-0.75$ and $0.06-1.25$, respectively, in the investigated frequency regime. MJK/PDMS7 is the most promising blend, due to a low dielectric loss factor of 0.05-0.125 (Fig. 8).

The behaviour of MJK/PDMS7 non-conductivity with different copolymer loadings is very promising, since no plateau regions are observed at low frequencies (Fig. 9). This implies that a blending method applied properly causes the successful formation of a discontinuous phase for PEG that creates nonconductive behaviour of the developed polymer in the PDMS elastomer and PDMS7-PEG blends at loadings of 5, 10, 15 and $20 \mathrm{wt} \%$. The conductivity of MJK/PDMS7 is consistent with respect to the MJK elastomer, which is non-conductive, as shown in Fig. 9.

The low dielectric loss factor and non-conductivity of MJK/PDMS7 for all investigated copolymer loadings indicates that the composites consist of PEG in discontinuous phases.

\section{Rheological properties of BPB}

To evaluate the effect of blending on mechanical properties, elastomers from MJK/PDMS7 with a 5-20 wt\% copolymer were rheologically characterised, as shown in Fig. 10. The storage modulus of MJK/PDMS7 with $20 \mathrm{wt} \%$ is relatively close to the storage modulus of silicone elastomer (MJK). In contrast, MJK/PDMS7 with 5 and $10 \mathrm{wt} \%$ is softer than the PDMS elastomer, with storage moduli being one-fold and three-fold lower than the storage modulus of MJK $\left(7 \times 10^{5}\right.$ $\mathrm{Pa})$. The blend of MJK/PDMS7 with $15 \mathrm{wt} \%$ is the stiffest, with $G^{\prime}=8 \times 10^{5} \mathrm{~Pa}$. Another important feature observed from Fig. 10 is the appearance of small relaxation peaks in the loss moduli for 15 and $20 \mathrm{wt} \%$. This is due to the transient nature of the PEG semi-crystalline phases acting as reinforcing domains.

All elastomers, however, do show to be well cross-linked and appear very elastic, and therefore they are suitable as soft dielectric elastomers.

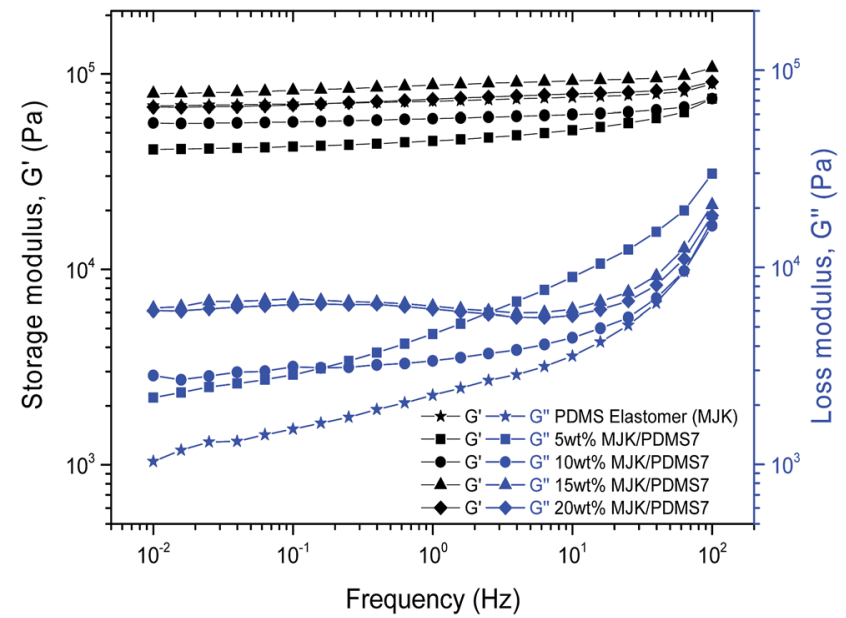

Fig. 10 The storage and loss moduli of MJK/PDMS7 (5-20 wt\% of PDMS7-PEG) at $23^{\circ} \mathrm{C}$.

\section{Dielectric breakdown $\left(E_{\mathrm{BD}}\right)$ strength}

Electrical breakdown and the influence of different PDMS7-PEG block copolymer loadings in MJK/PDMS7 on the Weibull parameters were investigated. The Weibull fits can be seen in Fig. 11. The Weibull $\beta$-parameter (slope of the dashed line in Fig. 11) decreases in line with an increasing MJK/PDMS7 wt\%, and it even increases at $20 \mathrm{wt} \%$. The $y$-axis (Fig. 11) was determined from the formula below:

$$
\ln [-\ln (1-F)]=\beta \ln \left(E_{\mathrm{BD}}\right)-\beta \ln (\eta)
$$

where $F$ and $E_{\mathrm{BD}}$ were the Weibull cumulative distribution function and electrical breakdown, respectively. The value of the Weibull location parameter $\eta$ was determined from $\ln [-\ln (1-F)]=63.2$.

Averaged and fitted electrical breakdown data for all the samples are presented in Table 3. MJK/PDMS7 with $5 \mathrm{wt} \%$ bears

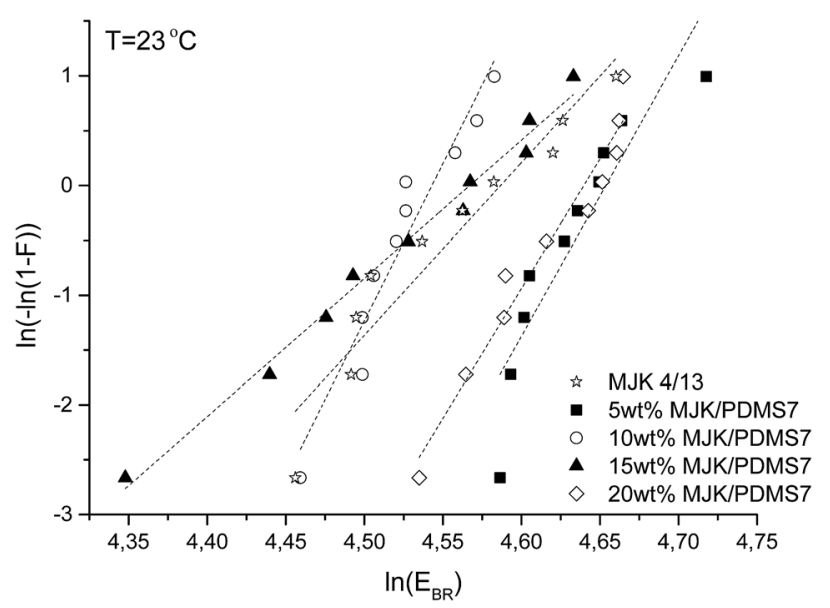

Fig. 11 Cumulative probability of failure of the PDMS elastomer (MJK) and MJK/PDMS7 with 5-20 wt\% of the PDMS7-PEG multiblock copolymer $\left(T=23^{\circ} \mathrm{C}\right)$. The dashed lines represent the linear fit line to the data. 
Table 3 Dielectric breakdown strength, Weibull parameters $\eta$ and $\beta$, and $R^{2}$ of linear fit for the pure silicone elastomer (MJK) and MJK/PDMS7 with 5-20 wt\% of the PDMS7-PEG multiblock copolymer

\begin{tabular}{|c|c|c|c|c|}
\hline MJK/PDMS7 & Dielectric breakdown $E_{\mathrm{BD}}\left(\mathrm{V} \mu \mathrm{m}^{-1}\right)$ & Weibull $\eta$-parameter & Weibull $\beta$-parameter & $R^{2}$ of linear fit \\
\hline MJK & $93 \pm 7$ & 98 & 17 & 0.92 \\
\hline 5 wt $\%$ & $103 \pm 4$ & 105 & 31 & 0.84 \\
\hline $15 \mathrm{wt} \%$ & $93 \pm 8$ & 96 & 13 & 0.99 \\
\hline $20 \mathrm{wt} \%$ & $101 \pm 5$ & 103 & 25 & 0.95 \\
\hline
\end{tabular}

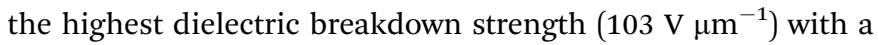

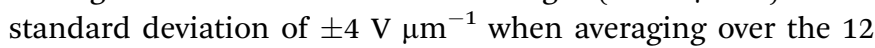
samples. All samples have an almost identical Weibull $\eta$ parameter and respective breakdown strengths.

Adding conductive particles usually destabilises the elastomer in respect to electrical breakdown, ${ }^{31}$ but in the composites investigated herein the conductive PEG clearly stabilises the elastomers, as the $\beta$ parameters for the composites are significantly larger - and thus the materials will be more electrically stable. This may be due to the charge-trapping effects of PEG. ${ }^{\mathbf{1 0}}$ The trapping effect probably decreases in line with increased loadings, and thus there is an optimum in the composition at which the electrical stabilisation is highest. The softest sample (5 wt\%) is furthermore very steep, and therefore the effect cannot be attributed to increased Young's moduli, as shown in Vudayagiri et al. ${ }^{28}$

\section{Figure of merit $\left(F_{\mathrm{OM}}\right)$}

One method which can be used to evaluate the actuation performance of the elastomer is by means of a figure of merit for dielectric elastomer actuators, $F_{\mathrm{OM}}(\mathrm{DEA})$, derived by SommerLarsen and Larsen: ${ }^{32}$

$$
F_{\mathrm{OM}}(\mathrm{DEA})=\frac{3 \varepsilon_{\mathrm{r}} \varepsilon_{0} E_{\mathrm{BD}}{ }^{2}}{Y}
$$

where $E_{\mathrm{BD}}$ is electrical breakdown, $\varepsilon_{0}$ is vacuum permittivity $\left(8.85 \times 10^{-12} \mathrm{~F} \mathrm{~m}^{-1}\right), \varepsilon_{\mathrm{r}}$ is relative permittivity and $Y$ is the Young's modulus.

The $F_{\mathrm{OM}}(\mathrm{DEA})$ for the MJK/PDMS7 samples was determined relative to the absolute value of the $F_{\text {OM }}(\mathrm{DEA})$ of Elastosil RT625 $\left(1.86 \times 10^{-24} \%\right)$, as reported by Vudayagiri et al. ${ }^{28}$ The normalised $F_{\text {OM}}(\mathrm{DEA})$ was calculated as:

$$
F_{\mathrm{OM}}(\mathrm{DEA})_{\text {Norm. }}=\frac{F_{\mathrm{OM}}(\mathrm{DEA})_{\text {Elastomer }}}{F_{\mathrm{OM}}(\mathrm{DEA})_{\mathrm{RT} 625}}
$$

The calculated figures of merit are shown in Table 4. The composite with $5 \mathrm{wt} \%$ has the highest normalised $F_{\text {OM }}$ (DEA) value at 17 , i.e. 17 times greater actuation than the reference elastomer. This composition is the best-performing elastomer amongst those investigated, due to the combination of high electrical breakdown strength, a low Young's modulus and relatively high dielectric permittivity.

\section{Contact angles of BPB}

The wettability of MJK/PDMS7 polymer blends was evaluated by static contact angle measurements. The nature of the
PDMS-PEG multiblock copolymer is known as one of the amphiphilic dynamic polymer chains. Similar to MJK/PDMS7, which consists of PDMS7-PEG block copolymers in the PDMS matrix, the trend on wettability leans toward amphiphilic behaviour. In Fig. 12, the contact angles of MJK/PDMS7 for different $w \mathrm{t} \%(5,10,15$ and 20) decline steeply for the first $20 \mathrm{~s}$ and are followed by a slight decrease until they are almost stable at the end of the time period. This indicates that the block copolymer in the polymer blends orients its polymer chains in order to achieve the lowest possible surface energy, since the copolymer comprises blocks of both hydrophobic PDMS and hydrophilic PEG. When the developed elastomer is exposed to air, the surface is controlled by the hydrophobic PDMS from the block copolymer and the matrix, but upon contact with water the chains re-orient and the PDMS blocks migrate back into the

Table 4 Normalised $F_{O M}(D E A)$ and Young's modulus $(Y)$ for MJK/PDMS7 with 5-20 wt\% of PDMS7-PEG multiblock copolymer

\begin{tabular}{llc}
\hline MJK/PDMS7 & Young's modulus, $Y^{a}(\mathrm{kPa})$ & Normalised $F_{\text {OM }}(\mathrm{DEA})$ \\
\hline $0 \mathrm{wt} \%(\mathrm{MJK})$ & 205 & 6.1 \\
$5 \mathrm{wt} \%$ & 123 & 17.2 \\
$10 \mathrm{wt} \%$ & 169 & 9.6 \\
$15 \mathrm{wt} \%$ & 238 & 8.0 \\
$20 \mathrm{wt} \%$ & 203 & 11.2
\end{tabular}

${ }^{a}$ Young's modulus calculated from $Y=3 G^{\prime}$.

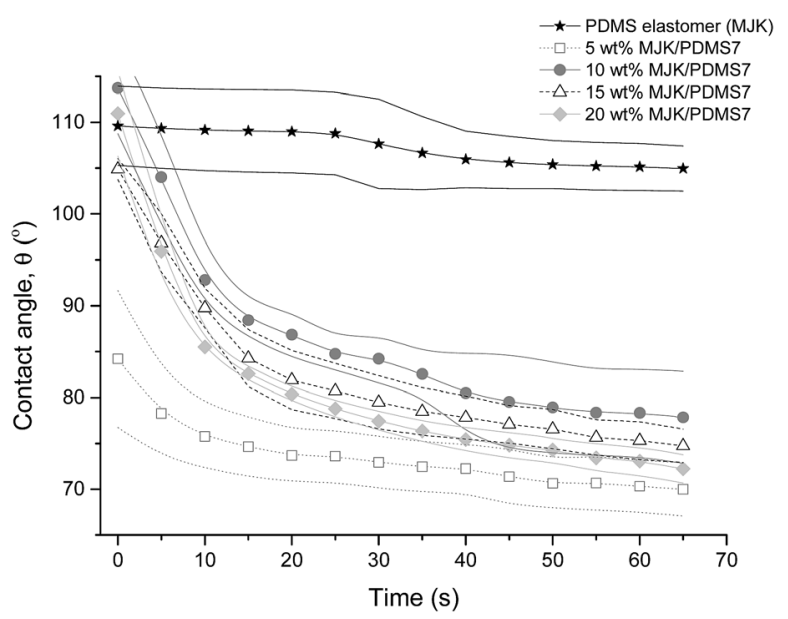

Fig. 12 MJK/PDMS7 contact angles (5-20 wt\% of PDMS7-PEG) at $23^{\circ} \mathrm{C}$. 

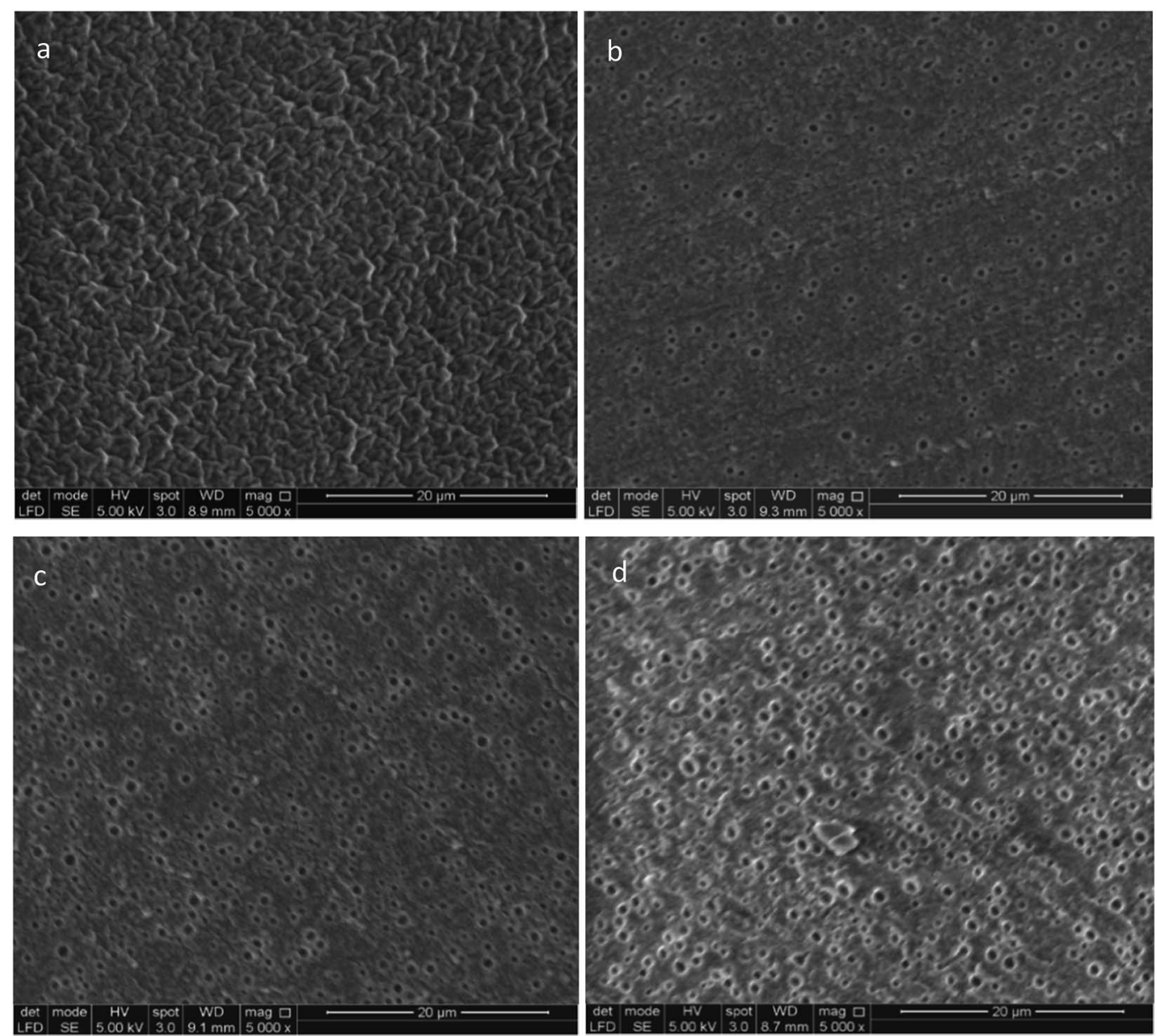

Fig. 13 SEM images of MJK/PDMS7 at: (a) 5 wt\% (b). 10 wt\% (c). 15 wt\% (d). 20 wt\%.

bulk material and are replaced by the more hydrophilic PEG blocks at the surface. ${ }^{23}$ This behaviour is confirmed by the contact angle measurement, where the rearrangement of the polymer chains accounts for the change in contact angle over time when a droplet of deionised water is dropped onto the top surface of the sample. Thus, classing the wettability of MJK/PDMS7 as amphiphilic is the result of incorporating the PDMS7-PEG multiblock copolymer in the network, since PEGs are well-known for their hydrophilic properties.

\section{SEM analysis}

In order to verify the hypothesised structure of the composites, the prepared films were investigated by SEM; the microscope pictures are shown in Fig. 13. For MJK/PDMS7 with 5 wt $\%$ copolymer loading, a rough surface is obtained. There are no visible PEG domains observed, and the composite appears homogeneous on the microscale. When the loading of the block copolymer increases from 10 to $20 \mathrm{wt} \%$, the microspherical domains become visible and the number of microspheres increases in line with an increased concentration of PEG. The domains were analysed using Image Processing and Analysis software (ImageJ). The domain sizes of visible spherical domains for MJK/PDMS7 at 10, 15 and $20 \mathrm{wt} \%$ are $1.3 \pm 0.2 \mu \mathrm{m}$,
$1.3 \pm 0.2 \mu \mathrm{m}$ and $1.6 \pm 0.2 \mu \mathrm{m}$, respectively. The observation of spherical domains is coherent with the samples from Liu et al.,$^{33}$ who observed pores on composite samples of PDMS and PEG etched with ethanol. ${ }^{33}$ The obtained morphologies indicate that the methodology of blending polymers creates the good dispersion of multiblock copolymers in a silicone network where the spherical domain size seems independent on concentration, as the chain length of the PEG was not a variable in this study. Since the composite with the lowest concentration of PEG possesses different morphology, and at the same time possesses the best overall properties for actuation and lifetime, it may be argued that the introduction of additional surfaces into the system is unfavourable, especially as these surfaces may increase permittivity but they also destabilise the elastomer.

\section{Conclusion}

A new composite elastomer, which has high relative and low permittivity, was successfully created from a binary system of polymer blends consisting of conducting PDMS7-PEG multiblock copolymer and non-conducting PDMS elastomer (MJK). The desired morphology (discontinuous phase of the block copolymer and continuous phase of PDMS) was successfully 
created in the blends, thereby indicating the development of non-conductive behaviour in the elastomer. Low copolymer loading is favourable, since it creates a homogeneous elastomer on the micro-scale which in turn facilitates a more electrically stable elastomer. Even though the PDMS7-PEG multiblock copolymer is conductive and has high loss permittivity, a good composite elastomer can be developed by incorporating the block copolymer into a silicone network at different wt $\%$ and by employing a proper mixing technique. The dielectric breakdown strengths for cross-linked MJK/PDMS7 polymer blends were relatively high, with values in the order of $100 \mathrm{~V} \mathrm{\mu m}^{-1}$. Finally, by integrating all the characterised parameters, i.e. Young's modulus, breakdown strength and relative permittivity, figures of merit for the dielectric elastomer actuation of the various MJK/PDMS7s were determined, and it was concluded that by incorporating low concentrations of PEG, actuation could be improved 17 -fold along with the extension to the lifetime of the dielectric elastomer.

\section{Acknowledgements}

The Malaysian Ministry of Education (MoE) and Universiti Tun Hussein Onn Malaysia (UTHM), as well as Innovationsfonden Danmark, are gratefully acknowledged for their funding.

\section{Notes and references}

1 S. Bauer, S. Bauer-Gogonea, I. Graz, M. Kaltenbrunner, C. Keplinger and R. Schwödiauer, Adv. Mater., 2014, 26, 149-162.

2 O. A. Araromi, I. Gavrilovich, J. Shintake, S. Rosset, M. Richard, V. Gass and H. R. Shea, IEEE ASME Trans. Mechatron., 2014, 20, 438-446.

3 I. A. Anderson, I. A. Ieropoulos, T. McKay, B. O'Brien and C. Melhuish, IEEE ASME Trans. Mechatron., 2011, 16, 107111.

4 T. G. McKay, B. M. O'Brien, E. P. Calius and I. A. Anderson, Appl. Phys. Lett., 2011, 98, 1-3.

5 R. Pelrine, R. Kornbluh, J. Joseph, R. Heydt, Q. Pei and S. Chiba, Mater. Sci. Eng., C, 2000, 11, 89-100.

6 J. L. Bigue, P. Chouinard, S. Proulx, G. Miron and J. S. Plante, Cansmart Work, 2014, 303-314.

7 X. Zhao and Z. Suo, Appl. Phys. Lett., 2007, 91, 24-27.

8 J. Plante and S. Dubowsky, Proc. SPIE, 2006, 6168, 61681J.

9 R. Kornbluh, R. Pelrine, Q. Pei, R. Heydt, S. Stanford, S. Oh and J. Eckerle, Proc. SPIE, 2002, 4698, 254-270.

10 S. Zakaria, P. H. F. Morshuis, M. Y. Benslimane, L. Yu and A. L. Skov, Smart Mater. Struct., 2015, 24, 1-10.

11 F. B. Madsen, A. E. Daugaard, C. Fleury, S. Hvilsted and A. L. Skov, RSC Adv., 2014, 4, 6939-6945.
12 P. Brochu and Q. Pei, Macromol. Rapid Commun., 2010, 31, 10-36.

13 F. B. Madsen, L. Yu, A. E. Daugaard, S. Hvilsted and A. L. Skov, RSC Adv., 2015, 5, 10254-10259.

14 B. Kussmaul, S. Risse, M. Wegener, M. Bluemke, J. Krause, J. Wagner, T. Feller, K. Clauberg, J. Hitzbleck, R. Gerhard and H. Krueger, Proc. SPIE, 2013, 8687, 86872S.

15 J. N. Lee, C. Park and G. M. Whitesides, Anal. Chem., 2003, 75, 6544-6554.

16 Y. Poojari and S. J. Clarson, J. Inorg. Organomet. Polym. Mater., 2009, 20, 46-52.

17 R. J. Sengwa, K. Kaur and R. Chaudhary, Polym. Int., 2000, 608, 599-608.

18 W. Tiejun, Q. I. Yingqun, X. U. Jingkun, H. U. Xiujie and C. Ping, Chin. Sci. Bull., 2003, 48, 2444-2445.

19 Y. Mai and A. Eisenberg, Chem. Soc. Rev., 2012, 41, 59695985.

20 A. L. Larsen and E. M. Terentjev, Macromolecules, 2006, 39, 9497-9507.

21 F. S. Bates and G. H. Fredrickson, Phys. Today, 1999, 52, 3238.

22 O. Gazit, R. Khalfin, Y. Cohen and R. Tannenbaum, J. Phys. Chem. C, 2009, 113, 576-583.

23 S. A. Klasner, E. C. Metto, G. T. Roman and C. T. Culbertson, Langmuir, 2009, 25, 10390-10396.

24 H. Jukarainen, S. Clarson, J. Seppala and L. Oy, Am. Chem. Soc., 2000, 729, 353-357.

25 M. P. Stevens, Polymer Chemistry (An Introduction), Oxford University Press, New York, 3rd edn, 1999.

26 A. L. Larsen, K. Hansen, P. Sommer-Larsen, O. Hassager, A. Bach, S. Ndoni and M. Jørgensen, Macromolecules, 2003, 36, 10063-10070.

27 S. M. G. Frankær, M. K. Jensen, A. G. Bejenariu and A. L. Skov, Rheol. Acta, 2012, 51, 559-567.

28 S. Vudayagiri, S. Zakaria, L. Yu, S. S. Hassouneh, M. Benslimane and A. L. Skov, Smart Mater. Struct., 2014, 23, 1-15.

29 M. Y. Benslimane, H. Kiil and M. J. Tryson, Polym. Int., 2010, 59, 415-421.

30 P. Ben Ishai, M. S. Talary, A. Caduff, E. Levy and Y. Feldman, Meas. Sci. Technol., 2013, 24, 1-21.

31 K. Goswami, A. E. Daugaard and A. L. Skov, RSC Adv., 2015, 5, 12792-12799.

32 P. Sommer-Larsen and A. L. Larsen, Proc. SPIE, 2004, 5385, 68-77.

33 H. Liu, L. Zhang, D. Yang, N. Ning, Y. Yu, L. Yao, B. Yan and M. Tian, J. Phys. D: Appl. Phys., 2012, 45, 1-9. 